This item was submitted to Loughborough's Research Repository by the author.

Items in Figshare are protected by copyright, with all rights reserved, unless otherwise indicated.

\title{
Optimisation of phase ratio in the triple jump using computer simulation
}

\section{PLEASE CITE THE PUBLISHED VERSION}

http://dx.doi.org/10.1016/j.humov.2015.12.012

\section{PUBLISHER}

(c) Elsevier

\section{VERSION}

AM (Accepted Manuscript)

\section{PUBLISHER STATEMENT}

This work is made available according to the conditions of the Creative Commons Attribution-NonCommercialNoDerivatives 4.0 International (CC BY-NC-ND 4.0) licence. Full details of this licence are available at: https://creativecommons.org/licenses/by-nc-nd/4.0/

\section{LICENCE}

CC BY-NC-ND 4.0

\section{REPOSITORY RECORD}

Allen, Sam, Mark King, and Fred Yeadon. 2016. "Optimisation of Phase Ratio in the Triple Jump Using Computer Simulation”. Loughborough University. https://hdl.handle.net/2134/20377. 


\title{
Optimisation of phase ratio in the triple jump using computer simulation
}

\author{
Sam J. Allen ${ }^{1}$, Mark A. King ${ }^{1}$, and M.R. (Fred) Yeadon ${ }^{1}$ \\ ${ }^{1}$ School of Sport, Exercise, and Health Sciences, Loughborough University, Loughborough, LE11 3TU, UK
}

\begin{abstract}
The triple jump is an athletic event comprising three phases in which the optimal proportion of each phase to the total distance jumped, termed the phase ratio, is unknown. This study used a whole-body torque-driven computer simulation model of all three phases of the triple jump to investigate optimal technique. The technique of the simulation model was optimised by varying torque generator activation parameters using a genetic algorithm in order to maximise total jump distance, resulting in a hop-dominated technique (35.7\%:30.8\%:33.6\%) and a distance of $14.05 \mathrm{~m}$. Optimisations were then run with penalties forcing the model to adopt hop and jump phases of 33\%, 34\%, 35\%, 36\%, and $37 \%$ of the optimised distance, resulting in total distances of: $13.79 \mathrm{~m}, 13.87 \mathrm{~m}$, $13.95 \mathrm{~m}, 14.05 \mathrm{~m}$, and $14.02 \mathrm{~m}$; and $14.01 \mathrm{~m}, 14.02 \mathrm{~m}, 13.97 \mathrm{~m}, 13.84 \mathrm{~m}$, and $13.67 \mathrm{~m}$ respectively. These results indicate that in this subject-specific case there is a plateau in optimum technique encompassing balanced and hop-dominated techniques, but that a jump-dominated technique is associated with a decrease in performance. Hopdominated techniques are associated with higher forces than jump-dominated techniques; therefore optimal phase ratio may be related to a combination of strength and approach velocity.
\end{abstract}

Keywords: computer simulation, triple jump, phase ratio, optimisation, technique,biomechanics

\section{INTRODUCTION}

The triple jump is an athletic event involving three consecutive phases during which athletes must distribute their 'effort' in order to maximise the total distance (Figure 1). The 'phase ratio' is the distances of each phase expressed as three percentages of the total distance. Triple jump techniques with respect to phase ratio have been defined as being: (a) hop-dominated - where the hop percentage is at least $2 \%$ greater than the next largest phase percentage; (b) jump-dominated where the jump percentage is at least $2 \%$ greater than the next largest phase percentage; and (c) balanced - where the largest phase percentage is less than $2 \%$ greater than the next largest phase percentage (Hay, 1992). There have been a number of attempts to determine the effect of phase ratio on triple jump performance using various approaches including: observations of elite jumpers (Miller and Hay, 1986; Hay, 1992; Hay, 1993; Hay, 1995; Hay, 1997; Hay, 1999; Song and Ryu, 2011); the differences between elite and novice jumpers (Simpson et al., 2007); statistical relationships between velocity tradeoffs during the contact phases ( $\mathrm{Yu}$ and Hay, 1996; Yu, 1999); and even an operations research approach (Brimberg et al., 2006).

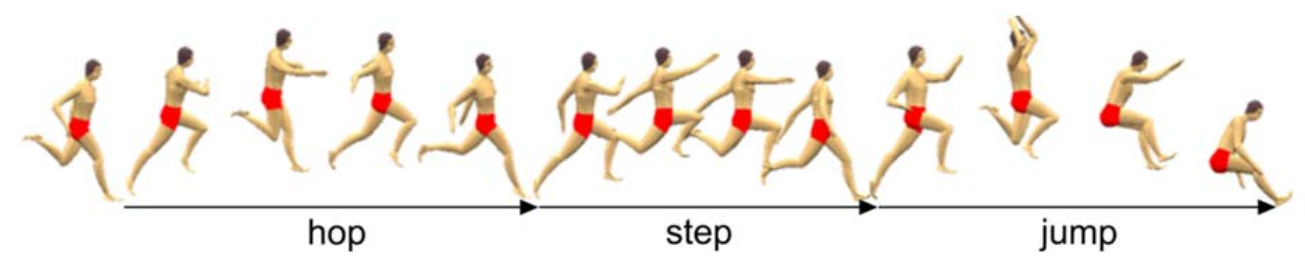

Figure 1. The three phases of the triple jump. 
Observations of triple jump performances can give misleading information regarding optimal phase ratio; since successful jumps can contain phases of similar lengths to less successful jumps, but make up smaller proportions of the distance jumped (Allen et al., 2013). For instance, consider two jumps of different total distance which had the same hop phase distances: in the longer jump the hop phase makes up a smaller percentage of the total distance than the shorter jump. Taken in isolation this would suggest that the athlete should reduce his or her hop phase distance, when in fact the hop phase distances were identical in the two performances. This effect may have contributed to previous observations that athletes tended to jump furthest when their hop phase percentages were relatively small when compared to their less successful jumps (Hay, 1999). With this in mind it is useful to consider absolute phase distances alongside percentages in order to understand the effect of employing phases of different lengths.

Statistical relationships take no account of mechanical limitations and can lead to optimal phase ratio predictions that are outside the ranges employed by elite performers (Yu and Hay, 1996; Brimberg et al., 2006). Yu and Hay (1996) predicted subject-specific optimal techniques that included hop and jump phase percentages of $28 \%$ and $42 \%$ respectively, and Brimberg et al. (2006) found optimal jump phase percentages of between $43 \%$ and $48 \%$. However Hay (1999) found the minimum hop percentage and maximum jump percentage across 41 analysed trials, representing the best jump for each of 41 subjects, during the Olympic Games and US Olympic Trials of 1996 to be $31.7 \%$ and $37.8 \%$ respectively, indicating that the predicted values may not be optimal in practice. Of these 41 subjects: $49 \%$ used a hop-dominated technique; $44 \%$ used a balanced technique; and $7 \%$ used a jumpdominated technique. Since elite performers employ a range of techniques it is possible that optimal phase-ratio is subject-specific, that some athletes are operating substantially below their potential, or that it has little or no influence on performance. Attempts have been made to optimise technique using computer simulations of the individual phases of the triple jump (Allen et al., 2010; Allen et al., 2013). These studies provided information about optimal techniques in each phase, although it was not possible to obtain a globally optimal phase ratio since phases were optimised individually.

It has been stated that the identification of the optimum phase ratio for an athlete, 'should take priority over all other problems of triple jump technique because, without a solution to this problem, all others must be considered in ignorance' (Hay, 1992). Despite this, no consensus has been reached as to whether optimum phase ratios for triple jumping exist, and if so, what they are and whether they are subjectspecific, or whether there is a single phase ratio which is optimal for all jumpers. The aim of this study was to determine the effect of adopting each of the three types of technique (hop-dominated, jump-dominated, and balanced) on total jump distance using a subject-specific whole-body forward dynamics computer simulation model of all three phases of the triple jump.

\section{METHODS}

\subsection{Data collection}

Kinematic and force data were gathered at the Loughborough University indoor High Performance Athletics Centre (HiPAC) from a male triple jumper of national standard (age: 22 years; mass: 72.6 kg; height: $1.82 \mathrm{~m}$; best performance: $14.35 \mathrm{~m}$ ). The study was carried out in accordance with the Loughborough University Ethical 
Advisory Committee guidelines. Forty-five $25 \mathrm{~mm}$ retroreflective markers were placed in positions on the body of the jumper in order that locations of joint centres could be determined. Eighteen Vicon MX cameras covered a volume of $18 \mathrm{~m} \times 2 \mathrm{~m}$ $\times 2.5 \mathrm{~m}$ spanning the last stride of the approach and the complete triple jump. Data were captured at $240 \mathrm{~Hz}$ during a single triple jump performance from an approach run of self-selected length. This resulted in an approach velocity of $8.1 \mathrm{~m} . \mathrm{s}^{-1}$ and triple jump distance of $13.00 \mathrm{~m}$, employing a balanced technique (35.5\%:30.4\%:34.1\%). Orientation, defined as the angle of the trunk in a global reference frame, and configuration angles were calculated by considering the joint centre coordinates in the sagittal plane. Quintic splines (Wood and Jennings, 1979) were fitted to the time histories of these angles.

\subsection{Simulation model}

A 13-segment planar torque-driven computer simulation model (Figure 2) was developed to investigate triple jumping technique (Allen et al., 2010; Allen et al., 2012). The 13 segments comprised: head + trunk, two upper arms, two forearms and hands, two thighs, two shanks, two 2-segment feet, with wobbling masses within the shanks, thighs, and torso. Non-linear spring-dampers connected the ends of the wobbling and fixed elements (Pain and Challis, 2001). Each foot had three points of contact with the ground at the heel, ball (metatarsophalangeal joint), and toe. The foot-ground interface was modelled using horizontal and vertical non-linear springdampers situated at the heel, ball, and toe of each foot (Allen et al., 2012). Viscoelastic parameters representing the wobbling masses and the foot-ground interface were previously determined via optimisation; these parameters were varied in order to minimise the differences between simulated ground reaction force and kinematic data, and equivalent performance data (Allen et al., 2013).

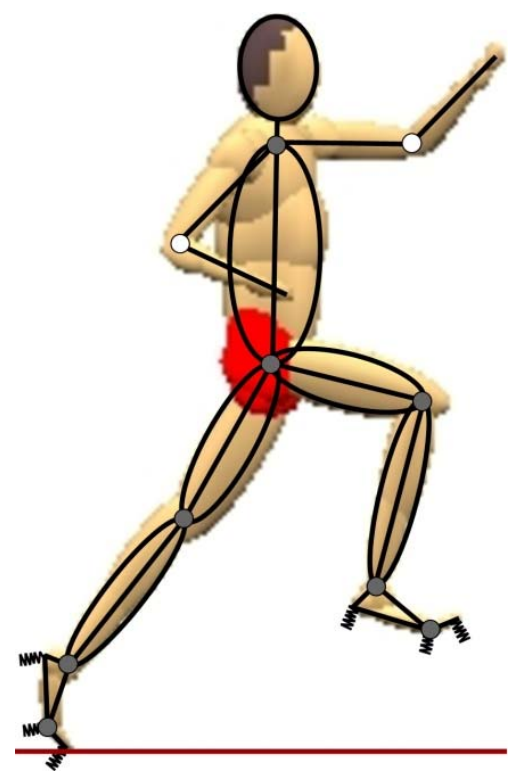

Figure 2. Thirteen-segment simulation model with wobbling masses within the shank, thigh, and trunk segments, torque drivers at the ball, ankle, knee, hip, and shoulder joints (grey circles), angle drivers at the elbow joints (white circles), and spring-dampers at three points on each foot. 


\subsection{Parameter determination}

Subject-specific torque and inertia parameters were calculated from measurements taken from the triple jumper. Maximal voluntary joint torque data were obtained assuming bilateral symmetry using an Isocom isovelocity dynamometer for flexion and extension of the ankle, knee, hip, and shoulder on the right hand side of the body (King et al., 2006). Ninety-five anthropometric measurements were taken along with body mass and used as input to the inertia model of Yeadon (1990) in order to calculate subject-specific segmental inertia parameters.

\subsection{Model evaluation}

The torque-driven model was evaluated by assessing how accurately a simulation could match performance data over one complete triple jump. This simulation was found by varying 246 parameters: 231 torque generator parameters (77 in each phase); and 15 initial kinematic conditions (the horizontal and vertical velocities of the centre of mass (CoM), the angular momentum about the CoM at the touchdown of the hop ground contact; and four initial angles in each phase: the orientation, and the hip, knee, and ankle angles of the stance leg), in order to minimise a difference function between simulation and performance data using a Genetic Algorithm (Carroll, 1996). The ground contact phases were simulated and the angular motion of the triple jumper in each airborne phase was estimated using performance data in order to calculate initial angular kinematics at the touchdown of each subsequent phase. The objective difference function for each matched torquedriven simulation was the root mean square (RMS) of six parts (Allen et al., 2010) averaged over the three phases (Eqn 1): percentage difference in horizontal velocity of the CoM at takeoff; percentage difference in vertical velocity of the CoM at takeoff; overall RMS difference in trunk orientation in degrees during ground contact; overall RMS difference in joint angles in degrees during ground contact; percentage absolute difference in time of contact; absolute difference in orientation at touchdown of the subsequent phase in degrees calculated as described by Allen et al. (2010).

$$
\operatorname{diff}=\frac{1}{3} \sum_{i=1}^{3} \sqrt{\frac{1}{6} \sum_{j=1}^{6} p_{i j}^{2}}
$$

where diff represents the overall difference, and $p_{i j}$ represents the six parts referred to above $(j=1,6)$ in each of the three phases of the triple jump $(i=1,3)$. In all cases $1^{\circ}$ was considered to be equivalent to $1 \%$ and objective difference function values were reported as percentages (Allen et al., 2010).

\subsection{Technique optimisation}

Initially the distance was maximised with no restrictions on phase percentages. Subsequently penalties were employed to force the model to adopt hop phases and jump phases representing 33\%,34\%,35\%,36\%, and 37\% of the unrestricted optimum distance, to determine the effect on the total distance jumped, leading to twelve optimisations in total. A Genetic Algorithm was used to maximise total jump distance by varying 243 parameters: 231 torque generator parameters (77 in each 
phase); and four initial angles in each phase as described above, giving 12 in total. Initial velocities were taken from the matched optimisation. Each phase distance ( $\left.d_{\text {phase }}\right)$ comprised three components (Figure 3$)$ : the takeoff distance $\left(d_{\text {takeoff }}\right)$, the flight distance $\left(d_{\text {flight }}\right)$, and the landing distance $\left(d_{\text {landing }}\right)$. The horizontal and vertical CoM position and velocity, and trunk orientation and whole-body angular momentum at takeoff from each phase were used in order to calculate the linear CoM velocity, and trunk orientation and angular velocity at the touchdown of the subsequent phase. As described above phase percentages do not represent equivalent distances for triple jumps of different lengths and therefore, in order to determine the effect of employing phases of different lengths, penalties were employed to constrain the simulations to adopt hop and jump phases within $\pm 0.005 \mathrm{~m}$ of $33 \%, 34 \%, 35 \%, 36 \%$ and $37 \%$ of the unrestricted optimised total triple jump distance.

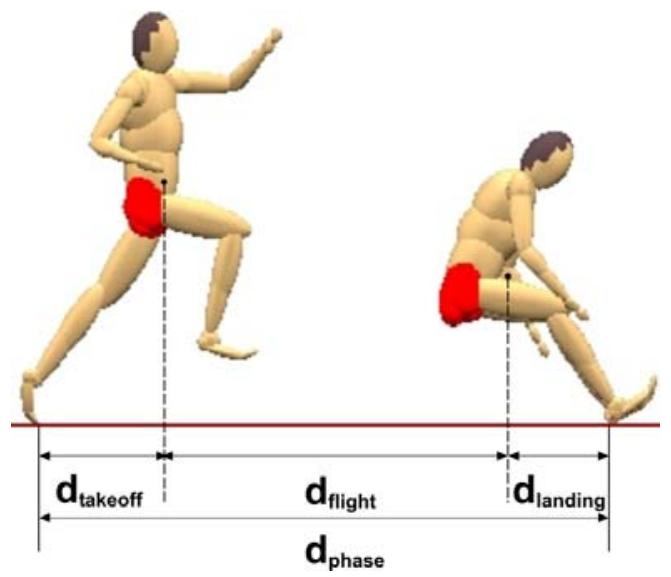

Figure 3. The three components of a phase distance $\left(d_{\text {phase }}\right)$ : the takeoff distance $\left(d_{\text {takeoff }}\right)$ is the horizontal distance from the toe of the stance leg to the CoM at takeoff; the flight distance $\left(d_{\text {flight }}\right)$ is the horizontal distance travelled by the CoM whilst the athlete is airborne; and the landing distance $\left(d_{\text {landing }}\right)$ is the horizontal distance from the CoM to the toe of the stance leg at touchdown, or in the case of the jump, the most posterior of the two heels at landing.

In the airborne phases orientation changes were assumed to be the same as those calculated from the measured performance configuration changes as described by Allen et al. (2010). The initial orientation was calculated from the takeoff orientation of the previous phase but was allowed to vary in each phase, since it was assumed that takeoff configurations and airborne motions different from the measured performance could lead to altered orientation changes during flight. The initial orientation of the hop phase was allowed to vary between $\pm 10^{\circ}$ from the matched performance, since it was assumed that the athlete could alter his orientation substantially during the approach run. The bounds on the variations in initial orientation in the step and jump phases were set to $\pm 20 \%$ of the magnitude of the changes in orientation that performance configuration changes produced in the previous flight phase. This led to bounds of $\pm 5^{\circ}$, and $\pm 2^{\circ}$ respectively from the landing orientations of the step and jump phases calculated from the previous phases. The initial ankle, knee, and hip angles were each allowed to vary by up to $\pm 5^{\circ}$ from the matched simulation.

The total distance of the optimised simulation was calculated along with the distance of each constituent phase. Technique is reported as per Hay's (1992) definition as either: hop-dominated; balanced; or jump-dominated. Each simulation was run for $100 \mathrm{~ms}$ after takeoff to ensure that the knee joint of the takeoff leg did 
not exceed anatomical limits early in the flight phase. Each optimisation necessitated around 20 million simulations; leading to over a quarter of a billion simulations in total.

\section{RESULTS}

\subsection{Model evaluation}

The matched simulation using the torque-driven model led to close correspondence with performance data, demonstrating sufficient complexity for subsequent optimisation of performance. The overall difference function value was $2.4 \%$, representing individual phase differences of 3.4\%,2.3\%, and $1.9 \%$ for the hop, step, and jump phases respectively. The matched simulation achieved a total jump distance of $12.67 \mathrm{~m}$ compared to the actual jump distance of $13.00 \mathrm{~m}$ (a difference of 2.5\%) and employed a balanced technique (Figures 4-8).

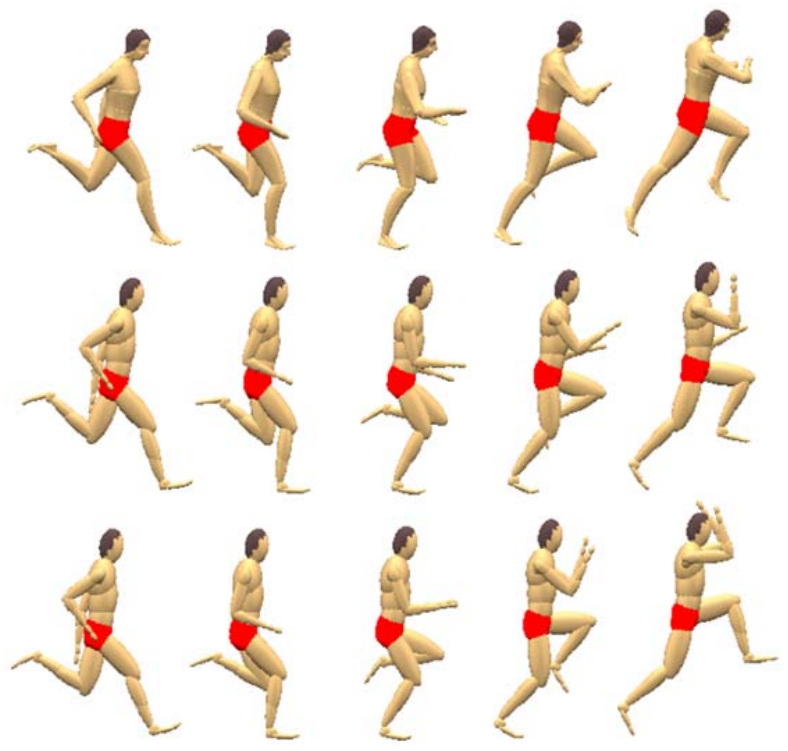

Figure 4. Performance (top) matched (middle), and optimised (bottom) simulations of the hop phase.
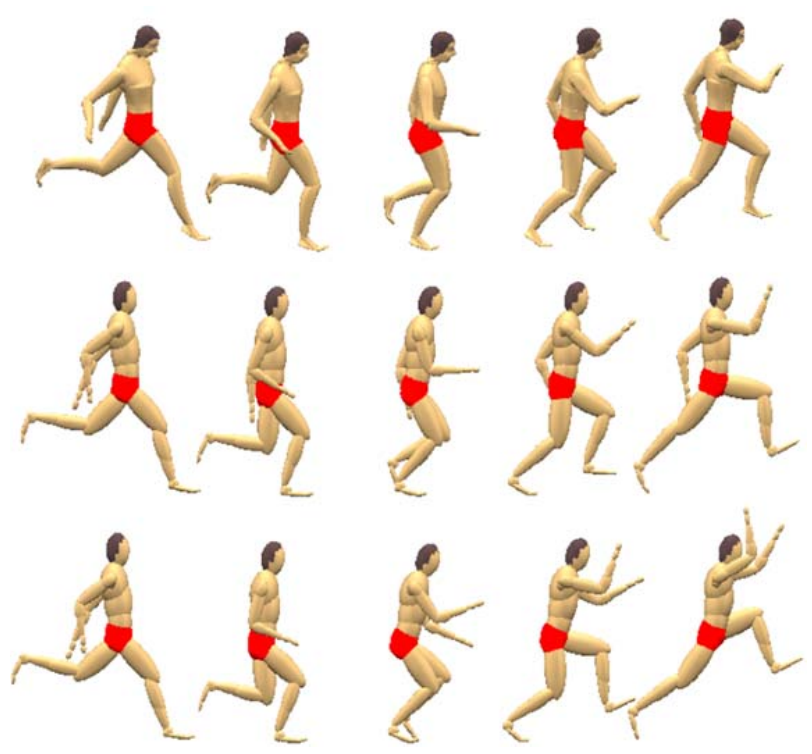

Figure 5. Performance (top), matched (middle), and optimised (bottom) simulations of the step phase. 


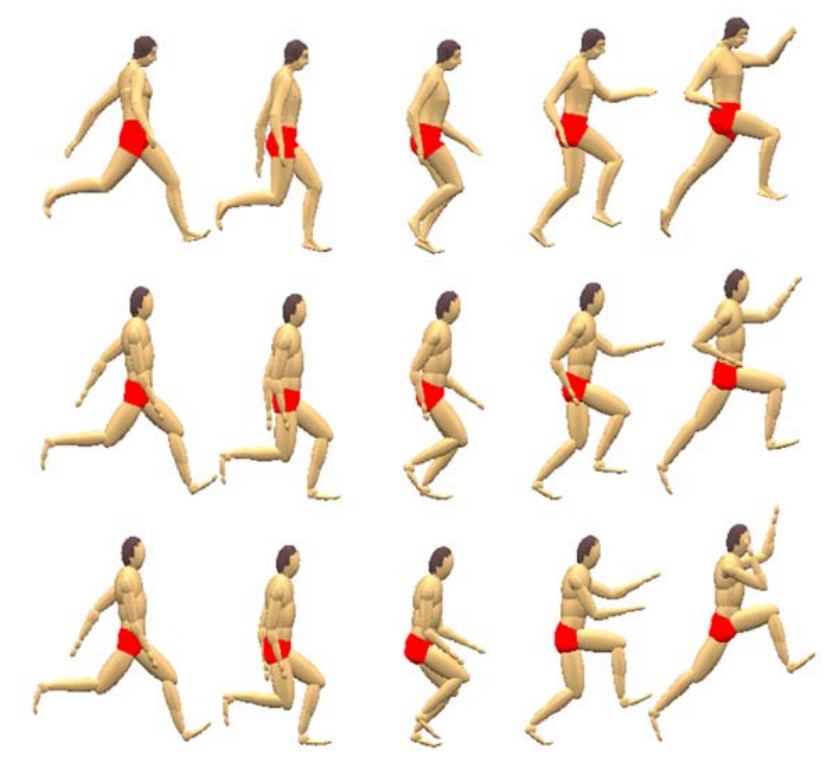

Figure 6. Performance (top), matched (middle), and optimised (bottom) simulations of the jump phase.

\subsection{Technique optimisation}

The optimisations of technique all resulted in improvements from the matched simulation. The overall optimisation of technique resulted in a total distance of 14.05 $\mathrm{m}$ and employed a hop-dominated technique (Figures 4-8). When the optimisation was forced to adopt hop phase distances representing 33\% (4.64 m), 34\% (4.78 m), $35 \%(4.92 \mathrm{~m}), 36 \%(5.06 \mathrm{~m})$, and $37 \%(5.20 \mathrm{~m})$ of the unrestricted optimum, this led to total distances of $13.79 \mathrm{~m}, 13.87 \mathrm{~m}, 13.95 \mathrm{~m}, 14.05 \mathrm{~m}$, and $14.02 \mathrm{~m}$ respectively (Figures 7 and 8). When the optimisation was forced to adopt equivalent jump distances this led to total distances of $14.01 \mathrm{~m}, 14.02 \mathrm{~m}, 13.97 \mathrm{~m}, 13.84 \mathrm{~m}$, and $13.67 \mathrm{~m}$ respectively (Figures 7 and 8 ).

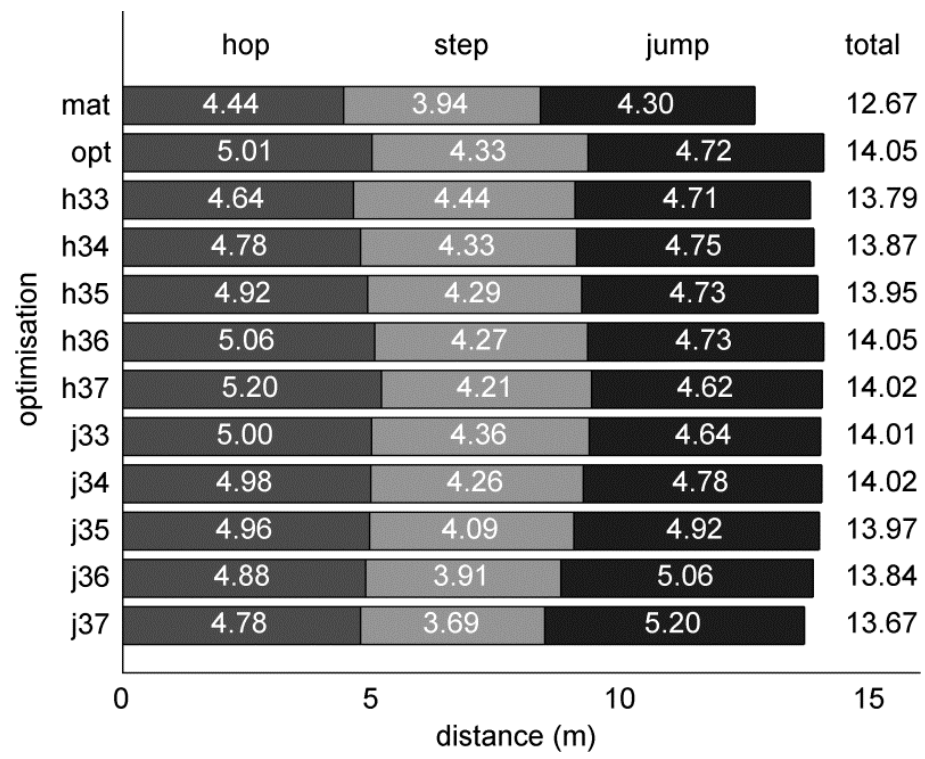

Figure 7. Optimised phase and overall distances. (Key: mat is the matched simulation; opt is the overall optimised simulation; and in the restricted simulations the letter represents the phase that was being restricted and the number represents the percentage of the overall optimum distance that phase was restricted to). 


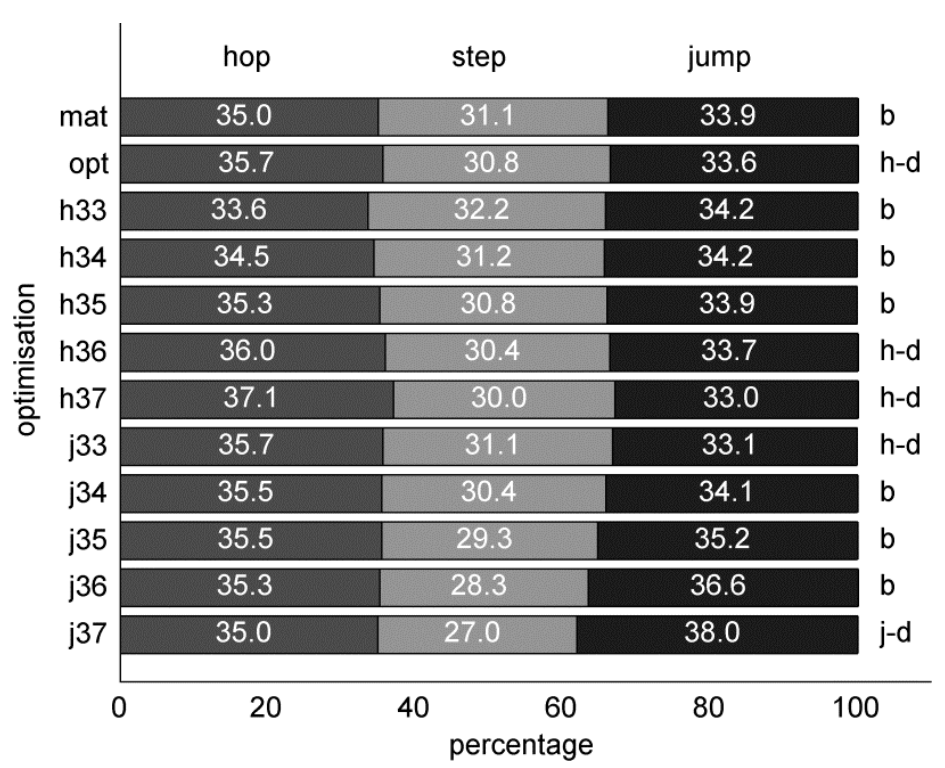

Figure 8. Optimised phase ratios. (Key: mat is the matched simulation; opt is the overall optimised simulation; and in the restricted simulations the letter represents the phase that was being restricted and the number represents the percentage of the overall optimum distance that phase was restricted to. Techniques are classified as balanced $-\mathrm{b}$, hop-dominated $-\mathrm{h}-\mathrm{d}$, or jump-dominated - j-d).

\section{DISCUSSION}

The results of the matching process indicated both that it was possible to simulate all three phases of the triple jump in a single process, and that the model was sufficiently complex to allow it to match the measured performance closely. The matched simulation achieved a jump distance only $2.5 \%$ less than the measured jump distance (12.67 m vs $13.00 \mathrm{~m}$ ) despite jump distance not being included explicitly in the objective difference function. Optimisation of technique resulted in the same kinematic elements noted by Allen et al., (2010): a symmetrical arm action; and a general trend towards greater extension of the joints of the stance leg and greater flexion of the free hip when compared with the matched simulation (Figures 4-6). Constrained optimisation of technique indicated that a hop-dominated technique was optimal for the athlete in this study but that deviating from this optimum technique towards a balanced or more hop-dominated technique did not result in the performance deteriorating substantially, indicating that there is a plateau in the optimal technique which encompasses a balanced and hop-dominated technique. Movement towards a jump-dominated technique however did result in a decrease in performance, indicating that this technique is sub-optimal for this athlete. The sensitivity of the overall distance to phase ratio, with the most jump-dominated technique leading to a distance reduction of less than $3 \%$ from the optimal technique, could be considered to be small, and if it were representative of triple jumpers as a whole might explain why a wide range of phase ratios are employed by jumpers in practice (German Athletics Federation, 2009; Hay, 1999; Korean Society of Sports Biomechanics, 2011). Nevertheless, in elite sport $3 \%$ is a meaningful margin, and represented the difference between winning and finishing out of the medals in the men's and women's triple jump events at the last four Olympic Games, therefore operating close to optimum technique is essential for success.

The benefits of a hop-dominated technique lie in the velocity tradeoffs during each ground contact phase. A unit gain in vertical velocity during the hop phase is more 
profitable than a unit gain in the jump phase; since the horizontal velocity is higher at this point it will result in a greater phase distance increase. It has been shown previously that, when technique is optimised, the loss in horizontal velocity during each ground contact phase is determined by the vertical takeoff velocity of that phase, and is largely independent of the vertical velocity at touchdown (Allen et al., 2013). Therefore a hop-dominated technique involving a high vertical velocity at the touchdown of the step does not incur great losses in horizontal velocity during the step ground contact. A high vertical velocity at the touchdown of the step ground contact does limit the vertical velocity at the takeoff of the step, and hence limits the phase distance. However the benefit of this is a low vertical velocity and relatively high horizontal velocity at the touchdown of the jump phase, which then still allows for a relatively long jump phase. In a jump-dominated technique the requirement is that the horizontal velocity is kept high enough, and the vertical velocity low enough, at the touchdown of the jump phase in order that the jump phase can be suitably long. This is achieved by limiting the vertical takeoff velocities, and hence the lengths, of the previous two phases. In doing this the jumper does not benefit from large gains in vertical velocity when the horizontal velocity is highest at the touchdown of the hop phase, and the gains achieved during the jump phase do not compensate for this, despite the lower height of the centre of mass at landing.

Given that there seems to be a plateau in optimal technique incorporating balanced and hop-dominated techniques (Figures 7 and 8), advising this athlete to utilise a moderately hop-dominated technique (36\%:30\%:34\%) would ensure that small deviations in either direction would result in approximately equivalent outcomes. In practice, however, the difficulty obviously comes in deciding what overall distance the $36 \%$ hop phase should be a proportion of, assuming the athlete has not yet achieved his optimum.

The results of this study are in line with the distribution of techniques observed in elite performers by Hay (1999): 49\% using a hop-dominated technique; $44 \%$ using a balanced technique; and $7 \%$ using a jump-dominated technique. However previous studies have indicated that at least two of the last four men's world records were set using a jump-dominated technique (Hay, 1999), and that perhaps the current world record (set in 1995) was also jump-dominated, although reliable data for this is not available (Hay, 1999). This may seem surprising given that the results of the optimisations in this study indicate that a jump-dominated technique is suboptimal. However, it has been proposed that the potential benefit of the jumpdominated technique is that it allows athletes to approach faster than they otherwise would be capable of (Hay, 1995). Optimisations in this study employed an approach velocity $\left(8.1{\left.\mathrm{~m} . \mathrm{s}^{-1}\right)}^{-}\right.$below that typically seen in elite performers $\left(9.5-10.5 \mathrm{~m} . \mathrm{s}^{-1}\right)$. So it is possible that a different optimum technique would be found for higher approach velocities. A balanced or hop-dominated technique may be harder to execute at higher approach velocities due to difficulties in controlling somersault rotation associated with the generation of high vertical velocity in the hop phase (Herzog, 1986). Also briefer stance phases during which to create vertical impulse will necessitate greater ground reaction forces, thus strength limitations may become apparent at higher approach velocities. When comparing the ground reaction forces from the most hop-dominated and jump-dominated optimised techniques it can be seen that the peak forces were highest in the hop-dominated technique during the step ground contact, and lowest in the same ground contact for the jump-dominated technique, whilst remaining similar between the two techniques across the other two ground contacts (Figure 9). The cause of these differences lies in the vertical 
velocity and body configuration at the touchdown of the step stance phase; in the hop-dominated technique the COM is lower due to a more shallow plant angle than in the jump-dominated technique. This led to a greater vertical velocity at the touchdown of the step stance phase $\left(-3.07 \mathrm{~m} . \mathrm{s}^{-1} \mathrm{vs}-2.68 \mathrm{~m} . \mathrm{s}^{-1}\right)$, despite a similar vertical impulse between the two techniques during the hop stance phase, resulting in higher impact forces. In addition to an increase in vertical velocity at touchdown, an increased plant angle also typically results in higher impact forces due to the more vertically oriented path of the COM as it pivots around the foot. Had the athlete approached faster, it is possible that he would not have been able to generate the impulses required to achieve a hop-dominated technique during the briefer stance phases that would have resulted, since it would have necessitated even higher forces, therefore a more balanced or jump-dominated technique might have been optimal.
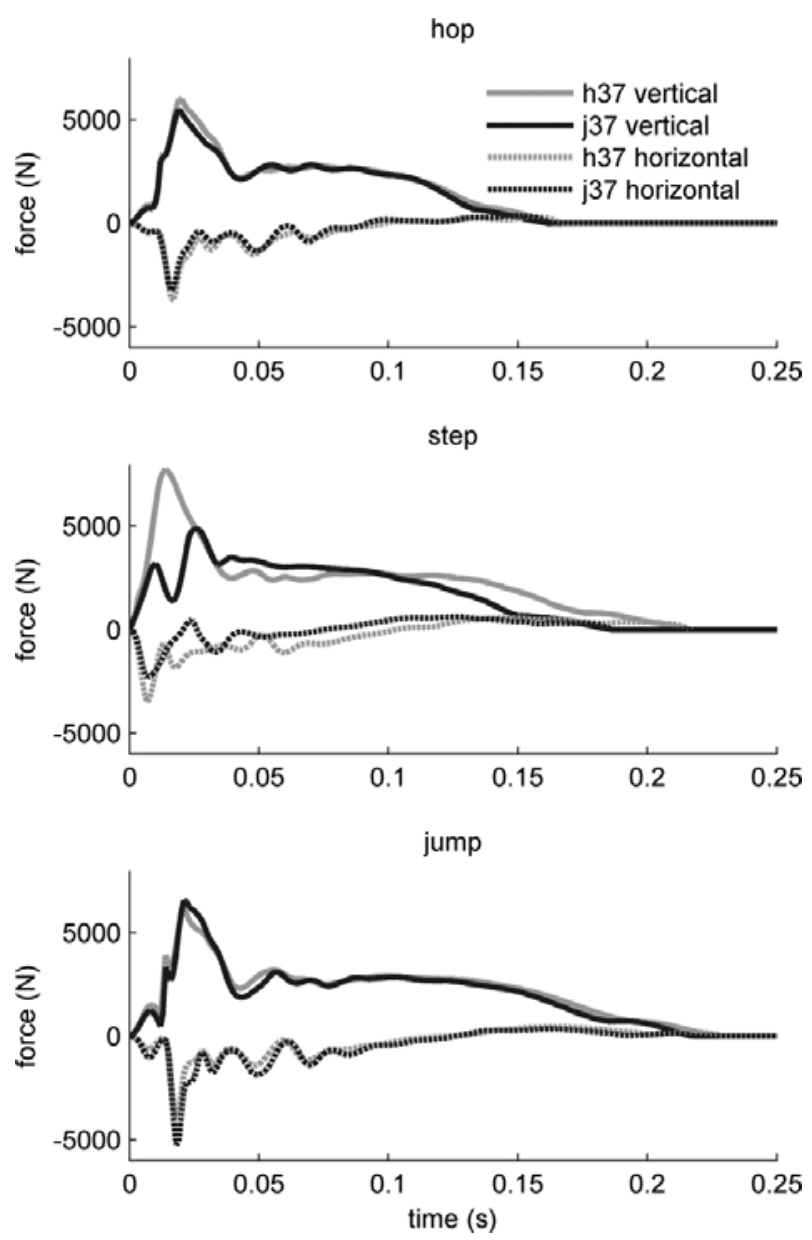

Figure 9. Horizontal and vertical ground reaction force time histories from each phase of the most hop and jump-dominated optimisations. (Key: h37 and j37 indicate the optimisations with hop and jump phases of $37 \%$ of the overall optimum distance respectively).

Athletes employing balanced or hop-dominated techniques may be either incapable of approaching at higher velocities, or may have reduced their approach velocity accordingly. Nonetheless, there is some evidence to suggest that the longest triple jump of all time, $18.43 \mathrm{~m}$ by the current world record holder Jonathan Edwards, comprised a balanced technique (35.3\%:30.4\%:34.3\%) (British Athletics, 2007). This ratio does not take account of the position of the foot on the takeoff board, so it is likely that the actual hop phase percentage was marginally greater still, 
and the other phase percentages marginally smaller. This was not ratified as a world record due to a wind speed measured at $0.4 \mathrm{~m} . \mathrm{s}^{-1}$ over the allowable limit, but is further than the current world record set by the same athlete $(18.29 \mathrm{~m})$ and indicates that it is possible that a balanced or hop-dominated technique may still be optimal at higher approach velocities if the athlete is able to coordinate his or her technique, and strong enough to generate the required vertical impulses.

It is worth noting that it was assumed that the strength of the model was bilaterally symmetrical; if an athlete had a slight strength discrepancy between limbs this could affect his or her optimal phase ratio. This study utilised a subject-specific model, which could be considered a limitation, since the optimal technique relates specifically to the subject. The main benefit of this approach is that model parameters can be obtained via direct measurement from the subject, thus the technique found via optimisation of the model is a realistic representation of the potential optimal technique of the subject. It could be argued that athletic techniques do not differ substantially between elite performers so it is likely that some generalisations can be made. However in order to generalise to a wider population with more confidence the effect of approach velocity and strength parameters on optimal phase ratio could be investigated, but was outside the scope of this study. The matched simulation distance was $2.5 \%$ different from the performance distance. The range of differences between optimised simulation distances was of the same order of magnitude $(\sim 3 \%)$; therefore this could lead to concerns that these differences were not valid. However our expectation is that, since the evaluation process indicated that the model was $97.5 \%$ correct in terms of the calculated distances, the model was therefore $97.5 \%$ correct in the differences between distances calculated during the subsequent optimisation procedure, which gives us confidence in the validity of our results.

\section{CONCLUSION}

In summary a hop-dominated technique was found to be optimal for the jumper in this study. When the model was forced to adopt a jump-dominated technique, overall distance was reduced by approximately 3\%. A hop-dominated technique is associated with higher forces than a jump-dominated technique; therefore it is possible that for athletes with higher approach speeds or reduced strength a jumpdominated technique may be optimal.

\section{REFERENCES}

Allen, S.J., King, M.A., Yeadon, M.R., 2010. Is a single or double arm technique more advantageous in triple jumping? Journal of Biomechanics, 43, 3156-3161.

Allen, S.J., King, M.A., Yeadon, M.R., 2012. Models incorporating pin joints are suitable for simulating performance but unsuitable for simulating internal loading. Journal of Biomechanics, 45, 1430-1436.

Allen, S.J., King, M.A., Yeadon, M.R., 2013. Trade-offs between horizontal and vertical velocities during triple jumping and the effect on phase distances. Journal of Biomechanics, 46, 979-983.

Brimberg, J., Hurley, B., Ladany, S.P., 2006. An operations research approach to the triple jump. International Journal of Sport Management and Marketing, 1, 208214. 
British Athletics: Jonathan Edwards [Internet] (2007). Birmingham: UK Athletics; [cited 2015 Apr 17]. Available from: http://www.britishathletics.org.uk/einspire/hall-of-fame-athletes/jonathan-edwards/.

Carroll, D.L., 1996. Chemical laser modeling with genetic algorithms. AIAA Journal, 34, 338-346.

German Athletics Federation, 2009. Biomechanical Analyses of Selected Events at the 12th IAAF World Championships in Athletics, Berlin, 15-23 August 2009 Triple Jump. Biomechanics Report World Championships 2009 Berlin, Deutscher Leichtathletik-Verband.

King, M.A., Wilson, C., Yeadon, M.R., 2006. Evaluation of a torque-driven model of jumping for height. Journal of Applied Biomechanics, 22, 264-274.

Hay, J.G., 1992. The biomechanics of the triple jump: a review. Journal of Sports Sciences, 10, 343-378.

Hay, J.G., 1993. Citius, altius, longius (faster, higher, longer): the biomechanics of jumping for distance. Journal of Biomechanics, 26, 7-21.

Hay, J.G., 1995. The case for a jump dominated technique in the triple jump. Track Coach, 132, 4214-4219.

Hay, J.G., 1997. Phase distances, percentages, and techniques in the men's triple jump at the 1996 U.S. Olympic trials. Track Coach, 139, 4435-4442.

Hay, J.G., 1999. Effort distribution and performance of Olympic triple jumpers. Journal of Applied Biomechanics, 15, 36-51.

Herzog, W., 1986. Maintenance of body orientation in the flight phase of long jumping. Medicine and Science in Sports and Exercise, 18, 231-241.

Korean Society of Sport Biomechanics, 2011. Biomechanics Research Project Report in the IAAF World Championships, Daegu 2011. 81-90.

Miller, J,A., Hay, J,G., 1986. Kinematics of a world record and other world-class performances in the triple jump. International Journal of Sports Biomechanics, 2, 272-288.

Pain, M.T.G., Challis, J.H., 2001. The role of the heel pad and shank soft tissue during impacts: a further resolution of a paradox. Journal of Biomechanics, 34, 327-333.

Simpson, S.E., Wilson, C., Kerwin, D.G., 2007. The changes in effort distribution from novice to experienced performers in the triple jump. XXV ISBS Symposium, Ouro Preto - Brazil.

Song, J.-H., Ryu, J.-K., 2011. Biomechanical analysis of the techniques and phase ratios of domestic elite triple jumpers. International Journal of Applied Sports Science, 23, 487-504.

Wood, G.A., Jennings, L.S., 1979. On the use of spline functions in data smoothing. Journal of Biomechanics, 12, 477-479.

Yeadon, M.R., 1990. The simulation of aerial movement-II. A mathematical inertia model of the human body. Journal of Biomechanics, 23, 67-74.

Yu, B., Hay, J.G., 1996. Optimum phase ratio in the triple jump. Journal of Biomechanics, 29, 1283-1289.

Yu, B., 1999. Horizontal-to-vertical velocity conversion in the triple jump. Journal of Sports Sciences, 17, 221-229. 